\title{
RATIONAL TORAL RANKS IN CERTAIN ALGEBRAS
}

\section{YASUSUKE KOTANI and TOSHIHIRO YAMAGUCHI}

\author{
Received 24 March 2004 and in revised form 17 September 2004
}

\begin{abstract}
We calculate the rational toral ranks of two spaces whose cohomologies are isomorphic and note that rational toral rank is a rational homotopy invariant but not a cohomology invariant.
\end{abstract}

2000 Mathematics Subject Classification: 55P62, 57S99.

1. Introduction. Let $\operatorname{rk}_{0}(Y)$ be the rational toral rank of a simply connected space $Y$, that is, the largest integer $r$ such that an $r$-torus $T^{r}=S^{1} \times \cdots \times S^{1}$ ( $r$-factors) can act continuously on a CW-complex which has the rational homotopy type of $Y$ with all its isotropy subgroups finite. For example, $\operatorname{rk}_{0}(Y)=1$ if $Y$ has the rational homotopy type of an odd-dimensional sphere $S^{2 n+1}$.

Let $\mathbb{Q}$ be the field of the rational numbers. For a finite-dimensional $\mathbb{Q}$-commutative graded algebra $A^{*}$ with $A^{0}=\mathbb{Q}$ and $A^{1}=0$, we put

$$
\begin{gathered}
2 \mathfrak{W}_{A^{*}}=\left\{\text { rational homotopy type of } Y \mid H^{*}(Y ; \mathbb{Q}) \cong A^{*}\right\}, \\
r_{A^{*}}=\left\{\operatorname{rk}_{0}(Y) \mid H^{*}(Y ; \mathbb{Q}) \cong A^{*}\right\},
\end{gathered}
$$

the set of rational toral ranks in $\mathfrak{W}_{A^{*}}$. For example, we see that if $A^{*}=A^{\text {even, }}$, then the Euler characteristic is nonzero, so there must be fixed points; hence, $r_{A^{*}}=\{0\}$. Note that $2 \mathfrak{b}_{A^{*}}$ and $\boldsymbol{r}_{A^{*}}$ are not empty sets since there exists the formal space whose cohomology is isomorphic to $A^{*}$ (see below), and that $r_{A^{*}}$ is at most finite even if $2 \boldsymbol{n}_{A^{*}}$ is infinite. In this paper, we calculate $r_{A^{*}}$ for certain commutative graded algebras $A^{*}$.

THEOREM 1.1. For the following four algebras $A^{*}$ :

(1) $A^{*} \cong H^{*}\left(S^{2} \vee S^{2} \vee S^{5} ; \mathbb{Q}\right)$,

(2) $A^{*} \cong H^{*}\left(\left(S^{3} \times S^{8}\right) \#\left(S^{3} \times S^{8}\right) ; \mathbb{Q}\right)$,

(3) $A^{*} \cong H^{*}\left(\left(S^{2} \vee S^{2}\right) \times S^{3} ; \mathbb{Q}\right)$,

(4) $A^{*} \cong H^{*}\left(\left(S^{2} \times S^{5}\right) \#\left(S^{2} \times S^{5}\right) ; \mathbb{Q}\right)$,

the rational toral ranks in $\mathfrak{W}_{A^{*}}$ are listed in Table 1.1, where $\mathfrak{W}_{A^{*}}=\{X, Y\}$ with a formal space $X$ and a nonformal space $Y$.

Here $\vee$ and \# denote a one point union (wedge) and a connected sum, respectively. For these $A^{*}$, we can check that $2 \mathfrak{W}_{A^{*}}$ is two points as in [5] or [6].

What do we know about the set $r_{A^{*}}$, namely, the function $\mathrm{rk}_{0}: 2 \mathfrak{W}_{A^{*}} \rightarrow\{0,1,2, \ldots\}$ ? For example, We consider the following questions.

QueSTION 1.2. Suppose that $A^{*}$ is a Poincaré duality algebra. Then, for $X, Y \in 2 \mathfrak{V}_{A^{*}}$, is $\operatorname{rk}_{0}(X) \leq \operatorname{rk}_{0}(Y)$ if $X$ is formal? 
TABLE 1.1. The rational toral ranks in $2 \mathfrak{W}_{A^{*}}$.

\begin{tabular}{c|cc}
\hline Algebra & $\mathrm{rk}_{0}(X)$ & $\mathrm{rk}_{0}(Y)$ \\
\hline$(1)$ & 0 & 0 \\
$(2)$ & 0 & 1 \\
$(3)$ & 1 & 0 \\
$(4)$ & 1 & 1 \\
\hline
\end{tabular}

A simply connected space $Y$ is called (rationally) elliptic if $\operatorname{dim} \pi_{*}(Y) \otimes \mathbb{Q}<\infty$ and $\operatorname{dim} H^{*}(Y ; \mathbb{Q})<\infty$.

QUESTION 1.3. For $X, Y \in 2 \mathfrak{W}_{A^{*}}$, is $\operatorname{rk}_{0}(X) \leq \operatorname{rk}_{0}(Y)$ if $Y$ is elliptic?

QUESTION 1.4. Is $r_{A^{*}}=\{a, a+1, \ldots, b-1, b\}$ for some integers $a \leq b$ ? Namely, are there no gaps in the sequence of integers of $r_{A^{*}}$ ?

Notice that, for our examples, the answer is positive for these questions.

For the proof of Theorem 1.1, we use the Sullivan minimal model $M(Y)$ of a simply connected space $Y$ of finite type. It is a free $\mathbb{Q}$-commutative differential graded algebra (d.g.a.) $(\wedge V, d)$ with a $\mathbb{Q}$-graded vector space $V=\bigoplus_{i>1} V^{i}$, where $\operatorname{dim} V^{i}<$ $\infty$ and a minimal differential, that is, $d\left(V^{i}\right) \subset\left(\wedge^{+} V \cdot \wedge^{+} V\right)^{i+1}$ and $d \circ d=0$. Here $\wedge V=$ (the $\mathbb{Q}$-polynomial algebra over $\left.V^{\text {even }}\right) \otimes\left(\right.$ the $\mathbb{Q}$-exterior algebra over $V^{\text {odd }}$ ) and $\wedge^{+} V$ is the ideal of $\wedge V$ generated by elements of positive degree. Denote the degree of an element $x$ of a graded algebra as $|x|$. Then $x y=(-1)^{|x||y|} y x$ and $d(x y)=$ $d(x) y+(-1)^{|x|} x d(y)$. Notice that $M(Y)$ determines the rational homotopy type of $Y$. See [3] for a general introduction and notation: for example, for the notion of KoszulSullivan (KS) extension. Especially note that $H^{*}(M(Y)) \cong H^{*}(Y ; \mathbb{Q})$ and a space $Y$ is said to be formal if there is a d.g.a. map $M(Y) \rightarrow\left(H^{*}(Y ; \mathbb{Q}), 0\right)$ which induces an isomorphism of cohomologies. The formal minimal model $M_{A^{*}}$ is constructed by a free commutative resolution of the algebra $A^{*}[5]$. Throughout this paper, $\mathbb{Q}\langle x, y, \ldots\rangle$ denotes the $\mathbb{Q}$-graded vector space generated by $\{x, y, \ldots\}$.

2. Preliminaries. Let $Y$ be a simply connected space of finite type with minimal model $M(Y)=(\wedge V, d)$. If an $r$-torus $T^{r}$ acts on $Y$, there is a KS extension, with $\left|t_{i}\right|=2$ for $i=1, \ldots, r$,

$$
\left(\mathbb{Q}\left[t_{1}, \ldots, t_{r}\right], 0\right) \longrightarrow\left(\mathbb{Q}\left[t_{1}, \ldots, t_{r}\right] \otimes \wedge V, D\right) \longrightarrow(\wedge V, d),
$$

which is induced from the Borel fibration [2]

$$
Y \rightarrow E T^{r} \times_{T^{r}} Y \rightarrow B T^{r}
$$

In particular, the fact that (2.1) is a KS extension entails that, $D t_{i}=0$ and for $v \in V$, $D v \equiv d v$ modulo the ideal $\left(t_{1}, \ldots, t_{r}\right)$, that is,

$$
D v=d v+\sum_{i_{1}+\cdots+i_{r}>0} h_{i_{1}, \ldots, i_{r}} t_{1} i_{1} \cdots t_{r} i_{r}
$$

with $h_{i_{1}, \ldots, i_{r}} \in \wedge V$. The differential $D$ also satisfies $D \circ D=0$. 
Lemma 2.1 [4, Proposition 4.2]. Suppose that $\operatorname{dim} H^{*}(Y ; \mathbb{Q})<\infty$. Then, $\operatorname{rk}_{0}(Y) \geq r$ if and only if there is a KS extension (2.1) satisfying $\operatorname{dim} H^{*}\left(\mathbb{Q}\left[t_{1}, \ldots, t_{r}\right] \otimes \wedge V, D\right)<\infty$.

So we may try to construct inductively for $1, \ldots, i$, the KS extensions:

$$
\left(\mathbb{Q}\left[t_{i}\right], 0\right) \longrightarrow\left(\mathbb{Q}\left[t_{1}, \ldots, t_{i}\right] \otimes \wedge V, D_{i}\right) \longrightarrow\left(\mathbb{Q}\left[t_{1}, \ldots, t_{i-1}\right] \otimes \wedge V, D_{i-1}\right)
$$

satisfying $\operatorname{dim} H^{*}\left(\mathbb{Q}\left[t_{1}, \ldots, t_{i}\right] \otimes \wedge V, D\right)<\infty$ in general. In the following, we consider the particular case of $i=1$.

LEMmA 2.2. Suppose that $H^{n+2}(\wedge V, d)=0$ and $H^{n}(\mathbb{Q}[t] \otimes \wedge V, D)=\mathbb{Q}\left\langle\gamma_{1}, \ldots, \gamma_{m}\right\rangle$. Then, $H^{n+2}(\mathbb{Q}[t] \otimes \wedge V, D) \subset \mathbb{Q}\left\langle\gamma_{1} t, \ldots, \gamma_{m} t\right\rangle$. Moreover, if $H^{n+1}(\wedge V, d)=0$, then the inclusion is an equality.

Proof. Let $\alpha+\alpha^{\prime} t$ be a $D$-cocycle in $(\mathbb{Q}[t] \otimes \wedge V)^{n+2}$ with $\alpha \in(\wedge V)^{n+2}$ and $\alpha^{\prime} \in$ $(\mathbb{Q}[t] \otimes \wedge V)^{n}$. Then we have $D \alpha=-D\left(\alpha^{\prime}\right) t$, and consequently, $d \alpha=0$.

Since $H^{n+2}(\wedge V, d)=0$, there is an element $\beta \in(\wedge V)^{n+1}$ such that $d \beta=\alpha$. Let $D \beta=$ $\alpha+\alpha^{\prime \prime} t$ for some $\alpha^{\prime \prime} \in(\mathbb{Q}[t] \otimes \wedge V)^{n}$. Then, since

$$
0=D^{2} \beta=D \alpha+D\left(\alpha^{\prime \prime}\right) t=-D\left(\alpha^{\prime}-\alpha^{\prime \prime}\right) t
$$

we see that $\alpha^{\prime}-\alpha^{\prime \prime}$ is a $D$-cocycle in $(\mathbb{Q}[t] \otimes \wedge V)^{n}$.

Since $H^{n}(\mathbb{Q}[t] \otimes \wedge V, D)=\mathbb{Q}\left\langle\gamma_{1}, \ldots, \gamma_{m}\right\rangle$, we can denote $\alpha^{\prime}-\alpha^{\prime \prime}=c_{1} \gamma_{1}+\cdots+c_{m} \gamma_{m}+$ $D \beta^{\prime}$ for some $c_{1}, \ldots, c_{m} \in \mathbb{Q}$ and $\beta^{\prime} \in(\mathbb{Q}[t] \otimes \wedge V)^{n-1}$. Then we have

$$
\begin{aligned}
\alpha+\alpha^{\prime} t & =\alpha+\left(\alpha^{\prime \prime}+c_{1} \gamma_{1}+\cdots+c_{m} \gamma_{m}+D \beta^{\prime}\right) t \\
& =c_{1} \gamma_{1} t+\cdots+c_{m} \gamma_{m} t+D\left(\beta+\beta^{\prime} t\right) .
\end{aligned}
$$

Hence $\left[\alpha+\alpha^{\prime} t\right]=\left[c_{1} \gamma_{1} t+\cdots+c_{m} \gamma_{m} t\right]$ in $H^{n+2}(\mathbb{Q}[t] \otimes \wedge V, D)$. Thus we have $H^{n+2}(\mathbb{Q}[t] \otimes \wedge V, D) \subset \mathbb{Q}\left\langle\gamma_{1} t, \ldots, \gamma_{m} t\right\rangle$.

Suppose that $c_{1} \gamma_{1} t+\cdots+c_{m} \gamma_{m} t=D\left(\eta+\eta^{\prime} t\right)$ for some $\eta \in(\wedge V)^{n+1}$ and $\eta^{\prime} \in(\mathbb{Q}[t] \otimes$ $\wedge V)^{n-1}$. Then we have $d \eta=0$ since $d \eta \notin \operatorname{Ideal}(t)$. If $H^{n+1}(\wedge V, d)=0$, there is an element $\theta \in(\wedge V)^{n}$ such that $d \theta=\eta$. Let $D \theta=\eta+\eta^{\prime \prime} t$ for some $\eta^{\prime \prime} \in(\mathbb{Q}[t] \otimes \wedge V)^{n-1}$. Then we have

$$
\left(c_{1} \gamma_{1}+\cdots+c_{m} \gamma_{m}\right) t=D\left(\eta+\eta^{\prime} t\right)=D\left(D \theta-\eta^{\prime \prime} t+\eta^{\prime} t\right)=D\left(\eta^{\prime}-\eta^{\prime \prime}\right) t .
$$

However, $c_{1} \gamma_{1}+\cdots+c_{m} \gamma_{m} \notin \operatorname{Im} D$ unless $c_{1}=\cdots=c_{m}=0$. Thus, if $H^{n+1}(\wedge V, d)=0$, $\gamma_{1} t, \ldots, \gamma_{m} t$ are linearly independent in $H^{n+2}(\mathbb{Q}[t] \otimes \wedge V, D)$. 
A commutative graded algebra $A^{*}$ with $\operatorname{dim} A^{*}<\infty$ will be said to have formal dimension $n$ if $A^{n} \neq 0$ and $A^{i}=0$ for all $i>n$. For example, the formal dimensions of (1), (2), (3), and (4) are 5, 11, 5, and 7, respectively.

LEMmA $2.3\left[4\right.$, Lemma 5.4]. Suppose that $H^{*}(\wedge V, d)$ and $H^{*}(\mathbb{Q}[t] \otimes \wedge V, D)$ have formal dimensions $n$ and $n^{\prime}$, respectively. Then $n^{\prime}=n-1$. If one algebra satisfies Poincaré duality, so does the other.

From Lemma 2.1 the following corollary may be useful to estimate a rational toral rank to be nonzero.

Corollary 2.4. Suppose that $H^{*}(\wedge V, d)$ has formal dimension $n$. Then, $\operatorname{dim} H^{*}(\mathbb{Q}[t]$ $\otimes \wedge V, D)<\infty$ if and only if $H^{n}(\mathbb{Q}[t] \otimes \wedge V, D)=H^{n+1}(\mathbb{Q}[t] \otimes \wedge V, D)=0$.

Proof. The "if" part is proved as follows. Since $H^{n+2 i}(\wedge V, d)=0$ for $i>0$, we have $H^{n+2 i}(\mathbb{Q}[t] \otimes \wedge V, D)=0$ for $i \geq 0$ from Lemma 2.2. Similarly, since $H^{n+2 i-1}(\wedge V, d)=0$ for $i>0$, we have $H^{n+2 i-1}(\mathbb{Q}[t] \otimes \wedge V, D)=0$ for $i>0$ from Lemma 2.2. Hence we have $H^{n+i}(\mathbb{Q}[t] \otimes \wedge V, D)=0$ for $i \geq 0$, that is, $\operatorname{dim} H^{*}(\mathbb{Q}[t] \otimes \wedge V, D)<\infty$.

The "only if" part follows from Lemma 2.3.

Proposition 2.5. Suppose that $H^{*}(\wedge V, d)$ has formal dimension $n$ and $(\wedge Z, D)$ is a minimal d.g.a. Then $H^{*}(\wedge Z, D)$ has formal dimension $n-1$ and $Z^{\leq n}=\mathbb{Q}\langle t\rangle \oplus V^{\leq n}$ with $D \equiv d \bmod (t)$ on $V^{\leq n}$ if and only if $Z=\mathbb{Q}\langle t\rangle \oplus V$ and $D \equiv d \bmod (t)$, that is, there is a $K S$ extension

$$
(\mathbb{Q}[t], 0) \longrightarrow(\wedge Z, D)=(\mathbb{Q}[t] \otimes \wedge V, D) \longrightarrow(\wedge V, d)
$$

such that $\operatorname{dim} H^{*}(\mathbb{Q}[t] \otimes \wedge V, D)<\infty$.

Proof. The "if" part is obvious from Lemma 2.3.

Now we show the "only if" part. For some $k \geq n$, assume that $Z^{\leq k}=\mathbb{Q}\langle t\rangle \oplus V^{\leq k}$ with $D v \equiv d v \bmod (t)$ for $v \in V^{\leq k}$. Then an element in $H^{k+2}\left(\wedge Z^{\leq k}, D\right)$ can be written using $\left[\alpha+\alpha^{\prime} t\right]$ with $\alpha \in\left(\wedge V^{\leq k}\right)^{k+2}$ and $\alpha^{\prime} \in\left(\wedge Z^{\leq k}\right)^{k}$. Since $D\left(\alpha+\alpha^{\prime} t\right)=0$, we have $d \alpha=0$. Now we give a map

$$
\rho_{k+1}: H^{k+2}\left(\wedge Z^{\leq k}, D\right) \longrightarrow H^{k+2}\left(\wedge V^{\leq k}, d\right)
$$

where $\rho_{k+1}\left(\left[\alpha+\alpha^{\prime} t\right]\right)=[\alpha]$. It is well defined. Indeed, if $\left[\alpha_{1}+\alpha_{1}^{\prime} t\right]=\left[\alpha_{2}+\alpha_{2}^{\prime} t\right]$ in $H^{k+2}\left(\wedge Z^{\leq k}, D\right)$, then $\alpha_{1}+\alpha_{1}^{\prime} t=\alpha_{2}+\alpha_{2}^{\prime} t+D\left(\beta+\beta^{\prime} t\right)$ for some $\beta \in\left(\wedge V^{\leq k}\right)^{k+1}$ and $\beta^{\prime} \in\left(\wedge Z^{\leq k}\right)^{k-1}$. Let $D \beta=d \beta+\beta^{\prime \prime} t$. Then we have

$$
\left(\alpha_{1}-\alpha_{2}\right)+\left(\alpha_{1}^{\prime}-\alpha_{2}^{\prime}\right) t=d \beta+\left(\beta^{\prime \prime}+D\left(\beta^{\prime}\right)\right) t
$$

So $\alpha_{1}-\alpha_{2}=d \beta$. Hence $\left[\alpha_{1}\right]=\left[\alpha_{2}\right]$ in $H^{k+2}\left(\wedge V^{\leq k}, d\right)$.

Since $\rho_{k+1}$ is bijective, from the following paragraphs we see that $Z^{k+1}=V^{k+1}$ with $D v \equiv d v \bmod (t)$ for $v \in V^{k+1}$ from the construction of minimal d.g.a.'s such that $H^{>k}(\wedge Z, D)=H^{>k}(\wedge V, d)=0$. Thus we have inductively $Z=\mathbb{Q}\langle t\rangle \oplus V$ with $D v \equiv$ $d v \bmod (t)$ for $v \in V$. 
Now we show that $\rho_{k+1}$ is injective. Suppose that $\rho_{k+1}\left(\left[\alpha+\alpha^{\prime} t\right]\right)=[\alpha]=0$. Then there is an element $\beta \in\left(\wedge V^{\leq k}\right)^{k+1}$ such that $d \beta=\alpha$. Let $D \beta=\alpha+\alpha^{\prime \prime} t$. Since $D(\alpha+$ $\left.\alpha^{\prime} t\right)=0$ and $D\left(\alpha+\alpha^{\prime \prime} t\right)=D^{2} \beta=0$, we have $D\left(\alpha^{\prime}-\alpha^{\prime \prime}\right)=0$. Since $H^{k}\left(\wedge Z^{\leq k}, D\right)=0$, $\alpha^{\prime}-\alpha^{\prime \prime}=D \beta^{\prime}$ for some $\beta^{\prime} \in\left(\wedge Z^{\leq k}\right)^{k-1}$. Then we have

$$
\alpha+\alpha^{\prime} t=\alpha+\left(\alpha^{\prime \prime}+D \beta^{\prime}\right) t=D\left(\beta+\beta^{\prime} t\right)
$$

Hence $\left[\alpha+\alpha^{\prime} t\right]=0$.

Now we show that $\rho_{k+1}$ is surjective. Let $[\alpha] \in H^{k+2}\left(\wedge V^{\leq k}, d\right)$. Since $d \alpha=0$, we can denote $D \alpha=\gamma t$ with $\gamma \in\left(\wedge Z^{\leq k}\right)^{k+1}$. Since $H^{k+1}\left(\wedge Z^{\leq k}, D\right)=0, \gamma=D \eta$ for some $\eta \in\left(\wedge Z^{\leq k}\right)^{k}$. Then we have

$$
D(\alpha-\eta t)=D \alpha-D(\eta) t=\gamma t-\gamma t=0 .
$$

Hence there is an element $[\alpha-\eta t] \in H^{k+2}\left(\wedge Z^{\leq k}, d\right)$ such that $f([\alpha-\eta t])=[\alpha]$.

From Lemma 2.1, we have the following.

COROLLARY 2.6. Let $M(Y)=(\wedge V, d)$ with cohomology of formal dimension $n$. If there is a minimal d.g.a. $(\wedge Z, D)$ such that $H^{*}(\wedge Z, D)$ has formal dimension $n-1$ and $Z^{\leq n}=$ $\mathbb{Q}\langle t\rangle \oplus V^{\leq n}$ with $D \equiv d \bmod (t)$ on $V^{\leq n}$, then $M\left(E S^{1} \times_{S^{1}} Y\right) \cong(\wedge Z, D)$, that is, $\operatorname{rk}_{0}(Y) \geq 1$.

In the following, $X$ is formal and $Y$ is nonformal.

\section{Examples}

EXAMPLE 3.1. Let $X=S^{2} \vee S^{2} \vee S^{5}$. Then $\chi_{H}(X)=\sum_{i}(-1)^{i} \operatorname{dim} H^{i}(X ; \mathbb{Q})=2>0$. Recall

$$
\chi_{H}\left(E S^{1} \times_{S^{1}} X\right)=\chi_{H}(X) \cdot \chi_{H}\left(B S^{1}\right)
$$

for a Borel fibration $X \rightarrow E S^{1} \times_{S^{1}} X \rightarrow B S^{1}$. Since $\chi_{H}\left(B S^{1}\right)=\infty$ we have $\chi_{H}\left(E S^{1} \times_{S^{1}}\right.$ $X)=\infty$, that is, $\operatorname{dim} H^{*}\left(E S^{1} \times_{S^{1}} X ; \mathbb{Q}\right)=\infty$. From Lemma $2.1, \operatorname{rk}_{0}(X)=0$. By the same argument, we have $\operatorname{rk}_{0}(Y)=0$.

Note that $\chi_{H}(X)=\chi_{H}(Y)=0$ in (2), (3), and (4).

REMARK 3.2. Even if $X$ is a wedge of spaces, $\operatorname{rk}_{0}(X)$ may not be zero. For example, $M\left(S^{3} \vee S^{3} \vee S^{4}\right)=(\wedge V, d)=(\wedge(x, y, z, \ldots), d)$ with $|x|=|y|=3$ and $|z|=4$ and $d x=$ $d y=d z=0$. On the other hand, $M\left(S^{2} \vee S^{3}\right)^{\leq 4}=(\wedge Z, D)^{\leq 4}=(\wedge(t, x, y, z), D)$ with $|t|=$ 2, $D t=D x=0, D y=t^{2}$, and $D z=x t$. From Corollary 2.6, we have $\operatorname{rk}_{0}\left(S^{3} \vee S^{3} \vee S^{4}\right) \geq 1$.

EXAMPLE 3.3. Let $X=\left(S^{3} \times S^{8}\right) \#\left(S^{3} \times S^{8}\right)$. Then

$$
A^{*}=H^{*}(X ; \mathbb{Q})=\frac{\wedge(x, y) \otimes \mathbb{Q}[w, u]}{\left(x y, x u, x w-y u, y w, w^{2}, w u, u^{2}\right)}
$$

with $|x|=|y|=3,|w|=|u|=8$ and $X$ has the minimal model

$$
\left(\wedge V_{X}, d\right)=\left(\wedge\left(x, y, w, u, v_{1}, v_{2}, v_{3}, v_{4}, v_{5}, v_{6}, v_{7}, z_{1}, \ldots\right), d\right)
$$


with $\left|v_{1}\right|=5,\left|v_{2}\right|=\left|v_{3}\right|=\left|v_{4}\right|=10,\left|v_{5}\right|=\left|v_{6}\right|=\left|v_{7}\right|=15,\left|z_{1}\right|=7$ and $d x=d y=$ $d w=d u=0, d v_{1}=x y, d v_{2}=x u, d v_{3}=x w-y u, d v_{4}=y w, d v_{5}=w^{2}, d v_{6}=w u$, $d v_{7}=u^{2}, d z_{1}=x v_{1}, \ldots$

From $D \circ D=0$, we have $D x=D y=0, D u=\lambda x t^{3}$, and $D w=-\lambda y t^{3}$ for $\lambda \in \mathbb{Q}$. Assume $\operatorname{dim} H^{*}\left(\mathbb{Q}[t] \otimes \wedge V_{X}, D\right)<\infty$. From Lemma 2.3, $\lambda \neq 0$. Let $D v_{1}=x y+a t^{3}$ for $a \in$ $\mathbb{Q}$ and $D z_{1}=x v_{1}+h t$ for $h \in\left(\mathbb{Q}[t] \otimes \wedge V_{X}, D\right)^{6}$. Then $0=D^{2} z_{1}=-a x t^{3}+D(h) t$. But there is no element $h$ such that $D h=a x t^{2}$. Hence we have $a=0$. Since $H^{*}(X ; \mathbb{Q})$ satisfies Poincaré duality with formal dimension 11 , so does $H^{*}\left(\mathbb{Q}[t] \otimes \wedge V_{X}, D\right)$ with formal dimension 10 from Lemma 2.3. Since $H^{3}\left(\mathbb{Q}[y] \otimes \wedge V_{X}, D\right)=\mathbb{Q}\langle x, y\rangle$ and $H^{i}\left(\wedge V_{X}, d\right)=0$ for $4 \leq i \leq 7$, we have $H^{7}\left(\mathbb{Q}[t] \otimes \wedge V_{X}, D\right)=\mathbb{Q}\left\langle x t^{2}, y t^{2}\right\rangle$ from Lemma 2.2. But

$$
x \cdot x t^{2}=x \cdot y t^{2}=0
$$

in $H^{10}\left(\mathbb{Q}[t] \otimes \wedge V_{X}, D\right)$ since $a=0$. This contradicts Poincaré duality. Thus $\operatorname{dim} H^{*}(\mathbb{Q}[t] \otimes$ $\left.\wedge V_{X}, D\right)=\infty$. From Lemma 2.1, we have $\operatorname{rk}_{0}(X)=0$.

Let $M(Y)=\left(\wedge V_{Y}, d\right)=(\wedge(x, y, z), d)$ with $|x|=|y|=3,|z|=5$ and $d x=d y=0$, $d z=x y$. Then $H^{*}(Y ; \mathbb{Q}) \cong A^{*}$.

Put $D x=D y=0$ and $D z=x y+t^{3}$. Then $\operatorname{dim} H^{*}\left(\mathbb{Q}[t] \otimes \wedge V_{Y}, D\right)<\infty$. From Lemma 2.1, we have $\operatorname{rk}_{0}(Y) \geq 1$. Also for any $D$, we have $D x=D y=0$. Thus $\operatorname{dim} H^{*}\left(\mathbb{Q}\left[t_{1}\right.\right.$, $\left.\left.t_{2}\right] \otimes \wedge V_{Y}, D\right)=\infty$. From the case of $r=2$ in Lemma 2.1, we have $\operatorname{rk}_{0}(Y)=1$.

EXAMPLE 3.4. Let $X=\left(S^{2} \vee S^{2}\right) \times S^{3}$. Then $A^{*}=H^{*}(X ; \mathbb{Q})=\mathbb{Q}\left[x_{1}, x_{2}\right] \otimes \wedge(y) /\left(x_{1}^{2}, x_{1} x_{2}\right.$, $\left.x_{2}^{2}\right)$ with $\left|x_{i}\right|=2,|y|=3$. When $D=d$, except for $D y=t^{2},\left(\mathbb{Q}[t] \otimes \wedge V_{X}, D\right)$ is the minimal model of $\left(S^{2} \vee S^{2}\right) \times S^{2}$. Hence $\operatorname{rk}_{0}(X) \geq 1$. In general, if $D y=0,\left[x_{i} y\right] \neq$ $0 \in H^{5}\left(\mathbb{Q}[t] \otimes \wedge V_{X}, D\right)$, then $\operatorname{dim} H^{*}\left(\mathbb{Q}[t] \otimes \wedge V_{X}, D\right)=\infty$ from Lemma 2.2. If $D y \neq$ $0, H^{\text {odd }}\left(\mathbb{Q}[t] \otimes \wedge V_{X}, D\right)=0$ from Lemma 2.3. In each case, $\operatorname{dim} H^{*}\left(\mathbb{Q}\left[t_{1}, t_{2}\right] \otimes \wedge V_{X}, D\right)$ cannot be finite. From the case of $r=2$ in Lemma 2.1, we have $\operatorname{rk}_{0}(X)=1$.

Let $Y$ be the nonformal space with $H^{*}(Y ; \mathbb{Q}) \cong A^{*}$. Then $M(Y)=\left(\wedge V_{Y}, d\right)$ is given by

$$
V_{Y} \leq 5=\mathbb{Q}\left\langle x_{1}, x_{2}, y, z_{1}, z_{2}, z_{3}, u_{1}, u_{2}, v_{1}, v_{2}, v_{3}\right\rangle
$$

with $\left|x_{i}\right|=2,|y|=\left|z_{i}\right|=3,\left|u_{i}\right|=4,\left|v_{i}\right|=5$ and $d x_{1}=d x_{2}=d y=0, d z_{1}=x_{1}^{2}, d z_{2}=$ $x_{1} x_{2}, d z_{3}=x_{2}^{2}, d u_{1}=x_{1} z_{2}-x_{2} z_{1}, d u_{2}=x_{1} z_{3}-x_{2} z_{2}-x_{2} y, d v_{1}=x_{1} u_{1}-z_{1} z_{2}, d v_{2}=$ $x_{1} u_{2}+x_{2} u_{1}-z_{1} z_{3}+z_{2} y, d v_{3}=x_{2} u_{2}-z_{2} z_{3}+z_{3} y$. Here $H^{5}\left(\wedge V_{Y}, d\right)=\mathbb{Q}\left\langle x_{1} y, x_{2} y\right\rangle$.

Now we show that $t^{3} \neq 0$ in $H^{6}\left(\mathbb{Q}[t] \otimes \wedge V_{Y}, D\right)$. Let $D x_{1}=D x_{2}=0, D y=a x_{1} t+$ $b x_{2} t+c t^{2}$ for $a, b, c \in \mathbb{Q}$ and $D z_{i}=d z_{i}+a_{i} x_{1} t+b_{i} x_{2} t+c_{i} t^{2}$ for $a_{i}, b_{i}, c_{i} \in \mathbb{Q}$. Assume that $t^{3}=D\left(p x_{1} y+q x_{2} y+e y t+f z_{1} t+g z_{2} t+h z_{3} t\right)$ for some $p, q, e, f, g, h \in \mathbb{Q}$. Since the right-hand side is equal to

$$
\begin{aligned}
(p a+ & f) x_{1}^{2} t+(p b+q a+g) x_{1} x_{2} t+(q b+h) x_{2}^{2} t \\
& +\left(p c+e a+f a_{1}+g a_{2}+h a_{3}\right) x_{1} t^{2}+\left(q c+e b+f b_{1}+g b_{2}+h b_{3}\right) x_{2} t^{2} \\
& +\left(e c+f c_{1}+g c_{2}+h c_{3}\right) t^{3},
\end{aligned}
$$


we have

$$
\begin{gathered}
p c+e a-p a a_{1}-p b a_{2}-q a a_{2}-q b a_{3}=0, \\
q c+e b-p a b_{1}-p b b_{2}-q a b_{2}-q b b_{3}=0, \\
e c-p a c_{1}-p b c_{2}-q a c_{2}-q b c_{3}=1 .
\end{gathered}
$$

On the other hand, let $D u_{i}=d u_{i}+e_{i} y t+f_{i} z_{1} t+g_{i} z_{2} t+h_{i} z_{3} t$ for $e_{i}, f_{i}, g_{i}, h_{i} \in \mathbb{Q}$ and $D v_{i}=d v_{i}+l_{i} u_{1} t+m_{i} u_{2} t$ for $l_{i}, m_{i} \in \mathbb{Q}$. Since

$$
\begin{aligned}
& 0=D^{2} u_{1} \\
& =\left(a_{2}+f_{1}\right) x_{1}^{2} t+\left(b_{2}-a_{1}+g_{1}\right) x_{1} x_{2} t+\left(-b_{1}+h_{1}\right) x_{2}^{2} t \\
& +\left(c_{2}+e_{1} a+f_{1} a_{1}+g_{1} a_{2}+h_{1} a_{3}\right) x_{1} t^{2} \\
& +\left(-c_{1}+e_{1} b+f_{1} b_{1}+g_{1} b_{2}+h_{1} b_{3}\right) x_{2} t^{2} \\
& +\left(e_{1} c+f_{1} c_{1}+g_{1} c_{2}+h_{1} c_{3}\right) t^{3}, \\
& 0=D^{2} u_{2} \\
& =\left(a_{3}+f_{2}\right) x_{1}^{2} t+\left(b_{3}-a_{2}-a+g_{2}\right) x_{1} x_{2} t+\left(-b_{2}-b+h_{2}\right) x_{2}{ }^{2} t \\
& +\left(c_{3}+e_{2} a+f_{2} a_{1}+g_{2} a_{2}+h_{2} a_{3}\right) x_{1} t^{2} \\
& +\left(-c_{2}-c+e_{2} b+f_{2} b_{1}+g_{2} b_{2}+h_{2} b_{3}\right) x_{2} t^{2} \\
& +\left(e_{2} c+f_{2} c_{1}+g_{2} c_{2}+h_{2} c_{3}\right) t^{3}, \\
& 0=D^{2} v_{1} \\
& =e_{1} x_{1} y t+\left(f_{1}+a_{2}\right) x_{1} z_{1} t+\left(g_{1}-a_{1}+l_{1}\right) x_{1} z_{2} t+\left(h_{1}+m_{1}\right) x_{1} z_{3} t \\
& -m_{1} x_{2} y t+\left(b_{2}-l_{1}\right) x_{2} z_{1} t+\left(-b_{1}-m_{1}\right) x_{2} z_{2} t \\
& +\left(l_{1} e_{1}+m_{1} e_{2}\right) y t^{2}+\left(c_{2}+l_{1} f_{1}+m_{1} f_{2}\right) z_{1} t^{2} \\
& +\left(-c_{1}+l_{1} g_{1}+m_{1} g_{2}\right) z_{2} t^{2}+\left(l_{1} h_{1}+m_{1} h_{2}\right) z_{3} t^{2}, \\
& 0=D^{2} v_{2} \\
& =\left(e_{2}+a_{2}\right) x_{1} y t+\left(f_{2}+a_{3}\right) x_{1} z_{1} t \\
& +\left(g_{2}-a+l_{2}\right) x_{1} z_{2} t+\left(h_{2}-a_{1}+m_{2}\right) x_{1} z_{3} t \\
& +\left(e_{1}+b_{2}-m_{2}\right) x_{2} y t+\left(f_{1}+b_{3}-l_{2}\right) x_{2} z_{1} t \\
& +\left(g_{1}-b-m_{2}\right) x_{2} z_{2} t+\left(h_{1}-b_{1}\right) x_{2} z_{3} t \\
& +\left(c_{2}+l_{2} e_{1}+m_{2} e_{2}\right) y t^{2}+\left(c_{3}+l_{2} f_{1}+m_{2} f_{2}\right) z_{1} t^{2} \\
& +\left(-c+l_{2} g_{1}+m_{2} g_{2}\right) z_{2} t^{2}+\left(-c_{1}+l_{2} h_{1}+m_{2} h_{2}\right) z_{3} t^{2}, \\
& 0=D^{2} v_{3} \\
& =a_{3} x_{1} y t+\left(a_{3}+l_{3}\right) x_{1} z_{2} t+\left(-a_{2}-a+m_{3}\right) x_{1} z_{3} t \\
& +\left(e_{2}+b_{3}-m_{3}\right) x_{2} y t+\left(f_{2}-l_{3}\right) x_{2} z_{1} t \\
& +\left(g_{2}+b_{3}-m_{3}\right) x_{2} z_{2} t+\left(h_{2}-b_{2}-b\right) x_{2} z_{3} t \\
& +\left(c_{3}+l_{3} e_{1}+m_{3} e_{2}\right) y t^{2}+\left(l_{3} f_{1}+m_{3} f_{2}\right) z_{1} t^{2} \\
& +\left(c_{3}+l_{3} g_{1}+m_{3} g_{2}\right) z_{2} t^{2}+\left(-c_{2}-c+l_{3} h_{1}+m_{3} h_{2}\right) z_{3} t^{2} \text {, }
\end{aligned}
$$


we have

$$
\begin{gathered}
a=-2 a_{2}+b_{3}, \quad b=a_{1}-2 b_{2}, \quad c=-a_{1} a_{2}+a_{1} b_{3}-b_{2} b_{3}, \\
a_{3}=b_{1}=0, \quad c_{1}=\left(a_{1}-b_{2}\right) b_{2}, \quad c_{2}=a_{2} b_{2}, \quad c_{3}=-\left(a_{2}-b_{3}\right) a_{2} .
\end{gathered}
$$

Hence (3.7) will be

$$
\begin{gathered}
\left(-2 a_{2}+b_{3}\right)\left(e-p b_{2}-q a_{2}\right)=0, \\
\left(a_{1}-2 b_{2}\right)\left(e-p b_{2}-q a_{2}\right)=0, \\
\left(-a_{1} a_{2}+a_{1} b_{3}-b_{2} b_{3}\right)\left(e-p b_{2}-q a_{2}\right)=1,
\end{gathered}
$$

respectively. By (3.12), $e-p b_{2}-q a_{2} \neq 0$ and $-a_{1} a_{2}+a_{1} b_{3}-b_{2} b_{3} \neq 0$. Then, by (3.10) and (3.11), $b_{3}=2 a_{2}$ and $a_{1}=2 b_{2}$, respectively. But this contradicts $-a_{1} a_{2}+a_{1} b_{3}-$ $b_{2} b_{3} \neq 0$. Thus $t^{3} \neq 0$ in $H^{6}\left(\mathbb{Q}[t] \otimes \wedge V_{Y}, D\right)$.

Since $H^{*}\left(\wedge V_{Y}, d\right)$ has formal dimension 5, from Lemma 2.3, we have $\operatorname{dim} H^{*}(\mathbb{Q}[t] \otimes$ $\left.\wedge V_{Y}, D\right)=\infty$. From Lemma 2.1, we have $\operatorname{rk}_{0}(Y)=0$.

EXAMPLE 3.5. Let $X=\left(S^{2} \times S^{5}\right) \#\left(S^{2} \times S^{5}\right)$. Then

$$
A^{*}=H^{*}(X ; \mathbb{Q})=\frac{\mathbb{Q}\left[x_{1}, x_{2}\right] \otimes \wedge\left(y_{1}, y_{2}\right)}{\left(x_{1}^{2}, x_{1} x_{2}, x_{2}^{2}, x_{1} y_{1}-x_{2} y_{2}, x_{1} y_{2}, x_{2} y_{1}, y_{1} y_{2}\right)}
$$

with $\left|x_{i}\right|=2,\left|y_{i}\right|=5$ and $X$ has a minimal model $M(X)=M_{A^{*}}=\left(\wedge V_{X}, d\right)$ where

$$
V_{X}{ }^{\leq 7}=\mathbb{Q}\left\langle x_{1}, x_{2}, z_{1}, z_{2}, z_{3}, u_{1}, u_{2}, y_{1}, y_{2}, v_{1}, v_{2}, v_{3}, w_{1}, \ldots, w_{9}, s_{1}, \ldots, s_{18}\right\rangle
$$

with $\left|x_{i}\right|=2,\left|z_{i}\right|=3,\left|u_{i}\right|=4,\left|y_{i}\right|=\left|v_{i}\right|=5,\left|w_{i}\right|=6,\left|s_{i}\right|=7$ and

$$
\begin{gathered}
d x_{1}=d x_{2}=d y_{1}=d y_{2}=0, \\
d z_{1}=x_{1}^{2}, \quad d z_{2}=x_{1} x_{2}, \quad d z_{3}=x_{2}^{2}, \\
d u_{1}=x_{1} z_{2}-x_{2} z_{1}, \quad d u_{2}=x_{1} z_{3}-x_{2} z_{2}, \\
d v_{1}=x_{1} u_{1}-z_{1} z_{2}, \quad d v_{2}=x_{1} u_{2}+x_{2} u_{1}-z_{1} z_{3}, \quad d v_{3}=x_{2} u_{2}-z_{2} z_{3}, \\
d w_{1}=x_{1} y_{1}-x_{2} y_{2}, \quad d w_{2}=x_{1} y_{2}, d w_{3}=x_{2} y_{1}, \\
d w_{4}=x_{1} v_{1}-z_{1} u_{1}, \quad d w_{5}=x_{1} v_{2}-z_{1} u_{2}-z_{2} u_{1}, \quad d w_{6}=x_{1} v_{3}-z_{2} u_{2}, \\
d w_{7}=x_{2} v_{1}-z_{2} u_{1}, \quad d w_{8}=x_{2} v_{2}-z_{2} u_{2}-z_{3} u_{1}, \quad d w_{9}=x_{2} v_{3}-z_{3} u_{2}, \\
d s_{1}=x_{1} w_{1}-z_{1} y_{1}+z_{2} y_{2}, \quad d s_{2}=x_{1} w_{2}-z_{1} y_{2}, \quad d s_{3}=x_{1} w_{3}-z_{2} y_{1}, \\
d s_{4}=x_{1} w_{4}-z_{1} v_{1}, \quad d s_{5}=x_{1} w_{5}-z_{1} v_{2}+\frac{1}{2} u_{1}^{2},
\end{gathered}
$$




$$
\begin{gathered}
d s_{6}=x_{1} w_{6}+x_{1} w_{8}-z_{1} v_{3}-z_{2} v_{2}+u_{1} u_{2}, \quad d s_{7}=x_{1} w_{7}-x_{2} w_{4}+\frac{1}{2} u_{1}^{2}, \\
d s_{8}=x_{1} w_{8}-x_{2} w_{5}+u_{1} u_{2}, \quad d s_{9}=x_{1} w_{9}-x_{2} w_{6}+\frac{1}{2} u_{2}^{2}, \\
d s_{10}=x_{2} w_{1}-z_{2} y_{1}+z_{3} y_{2}, \quad d s_{11}=x_{2} w_{2}-z_{2} y_{2}, \quad d s_{12}=x_{2} w_{3}-z_{3} y_{1}, \\
d s_{13}=x_{2} w_{4}-z_{2} v_{1}-\frac{1}{2} u_{1}^{2}, \quad d s_{14}=x_{2} w_{5}+x_{2} w_{7}-z_{2} v_{2}-z_{3} v_{1}-u_{1} u_{2}, \\
d s_{15}=x_{2} w_{6}-z_{2} v_{3}, \quad d s_{16}=x_{2} w_{7}-x_{1} w_{6}+z_{1} v_{3}-z_{3} v_{1}-u_{1} u_{2}, \\
d s_{17}=x_{2} w_{8}-z_{3} v_{2}-\frac{1}{2} u_{2}^{2}, \quad d s_{18}=x_{2} w_{9}-z_{3} v_{3} .
\end{gathered}
$$

Let $(\wedge Z, D)$ be the formal minimal model $M_{B^{*}}$ for the Poincaré duality algebra

$$
B^{*}=\frac{\mathbb{Q}\left[t, x_{1}, x_{2}\right]}{\left(x_{1} t^{2}, x_{2} t^{2}, x_{1}^{2}+x_{2} t, x_{1} x_{2}-t^{2}, x_{2}^{2}+x_{1} t\right)}
$$

with $|t|=\left|x_{i}\right|=2$. Note $B^{*}$ has formal dimension 6 . Then

$$
Z^{\leq 7}=\mathbb{Q}\langle t\rangle \oplus V_{X} \leq 7
$$

with

$$
\begin{gathered}
D t=D x_{1}=D x_{2}=0, \quad D y_{1}=x_{2} t^{2}, \quad D y_{2}=x_{1} t^{2}, \\
D z_{1}=d z_{1}+x_{2} t, \quad D z_{2}=d z_{2}-t^{2}, \quad D z_{3}=d z_{3}+x_{1} t, \\
D u_{1}=d u_{1}+z_{3} t, \quad D u_{2}=d u_{2}-z_{1} t, \\
D v_{1}=d v_{1}-u_{2} t, \quad D v_{2}=d v_{2}, \quad D v_{3}=d v_{3}-u_{1} t, \\
D w_{1}=d w_{1}, \quad D w_{2}=d w_{2}+y_{1} t-z_{1} t^{2}, \quad D w_{3}=d w_{3}+y_{2} t-z_{3} t^{2}, \\
D w_{4}=d w_{4}+v_{2} t, \quad D w_{5}=d w_{5}+v_{3} t, \quad D w_{6}=d w_{6}+v_{1} t, \\
D w_{7}=d w_{7}+v_{3} t, \quad D w_{8}=d w_{8}+v_{1} t, \quad D w_{9}=d w_{9}+v_{2} t, \\
D s_{1}=d s_{1}+w_{3} t+u_{1} t^{2}, \quad D s_{2}=d s_{2}-w_{1} t, \quad D s_{3}=d s_{3}-w_{2} t+u_{2} t^{2}, \\
D s_{4}=d s_{4}-w_{5} t+w_{7} t, \quad D s_{5}=d s_{5}-w_{6} t+w_{8} t, \quad D s_{6}=d s_{6}-2 w_{4} t+w_{9} t, \\
D s_{7}=d s_{7}-w_{6} t+w_{8} t, \quad D s_{8}=d s_{8}-w_{4} t+w_{9} t, \quad D s_{9}=d s_{9}-w_{5} t+w_{7} t, \\
D s_{10}=d s_{10}-w_{2} t+u_{2} t^{2}, \quad D s_{11}=d s_{11}-w_{3} t-u_{1} t^{2}, \quad D s_{12}=d s_{12}+w_{1} t, \\
D s_{13}=d s_{13}-w_{8} t, \quad D s_{14}=d s_{14}+w_{4} t-2 w_{9} t, \quad D s_{15}=d s_{15}-w_{7} t, \\
D s_{16}=d s_{16}+2 w_{4} t-2 w_{9} t, \quad D s_{17}=d s_{17}+w_{5} t-w_{7} t, \quad D s_{18}=d s_{18}+w_{6} t-w_{8} t,
\end{gathered}
$$

that is, $D \equiv d \bmod (t)$ on $V_{X} \leq 7$. From Corollary 2.6, we have $\operatorname{rk}_{0}(X) \geq 1$. Also for any $D$ satisfying $\operatorname{dim} H^{*}\left(\mathbb{Q}[t] \otimes \wedge V_{X}, D\right)<\infty$, we see $H^{\text {odd }}\left(\mathbb{Q}[t] \otimes \wedge V_{X}, D\right)=0$ from Lemma 2.3. From the case of $r=2$ in Lemma 2.1, we have $\operatorname{rk}_{0}(X)=1$.

Let $M(Y)=\left(\wedge V_{Y}, d\right)=\left(\wedge\left(x_{1}, x_{2}, z_{1}, z_{2}, z_{3}\right), d\right)$ with $\left|x_{i}\right|=2,\left|z_{i}\right|=3$ and $d x_{1}=d x_{2}=$ $0, d z_{1}=x_{1}^{2}, d z_{2}=x_{1} x_{2}, d z_{3}=x_{2}^{2}$. Then $H^{*}(Y ; \mathbb{Q}) \cong A^{*}$. 
Put $D=d$ except for $D z_{2}=x_{1} x_{2}-t^{2}$. Then we have $\operatorname{dim} H^{*}\left(\mathbb{Q}[t] \otimes \wedge V_{Y}, D\right)<\infty$. From the case of $r=1$ in Lemma 2.1, $\operatorname{rk}_{0}(Y) \geq 1$. From [1], we have $\operatorname{rk}_{0}(Y)=1$. Indeed,

$$
\operatorname{rk}_{0}(Y) \leq-\chi_{\pi}(Y)=-\sum_{i}(-1)^{i} \operatorname{dim} \pi_{i}(Y) \otimes \mathbb{Q}=\operatorname{dim} V_{Y}^{\text {odd }}-\operatorname{dim} V_{Y}^{\text {even }}=1 .
$$

\section{REFERENCES}

[1] C. Allday and S. Halperin, Lie group actions on spaces of finite rank, Quart. J. Math. Oxford Ser. (2) 29 (1978), no. 113, 63-76.

[2] C. Allday and V. Puppe, Cohomological Methods in Transformation Groups, Cambridge Studies in Advanced Mathematics, vol. 32, Cambridge University Press, Cambridge, 1993.

[3] Y. Félix, S. Halperin, and J.-C. Thomas, Rational Homotopy Theory, Graduate Texts in Mathematics, vol. 205, Springer-Verlag, New York, 2001.

[4] S. Halperin, Rational homotopy and torus actions, Aspects of Topology, London Math. Soc. Lecture Note Ser., vol. 93, Cambridge University Press, Cambridge, 1985, pp. 293306.

[5] S. Halperin and J. Stasheff, Obstructions to homotopy equivalences, Adv. Math. 32 (1979), no. 3, 233-279.

[6] H. Shiga and T. Yamaguchi, The set of rational homotopy types with given cohomology algebra, Homology Homotopy Appl. 5 (2003), no. 1, 423-436.

Yasusuke Kotani: Department of Mathematics, Faculty of Science, Kochi University, Kochi 7808520, Japan

E-mail address: kotani@math.kochi-u.ac.jp

Toshihiro Yamaguchi: Department of Mathematics Education, Faculty of Education, Kochi University, Kochi 780-8520, Japan

E-mail address: tyamag@cc.kochi-u.ac.jp 


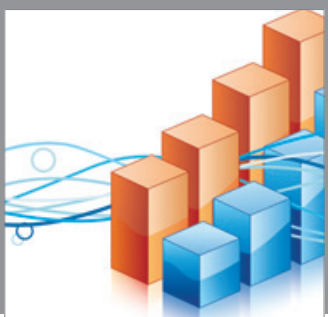

Advances in

Operations Research

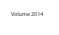

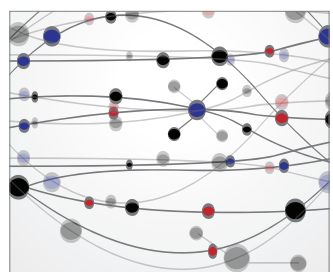

\section{The Scientific} World Journal
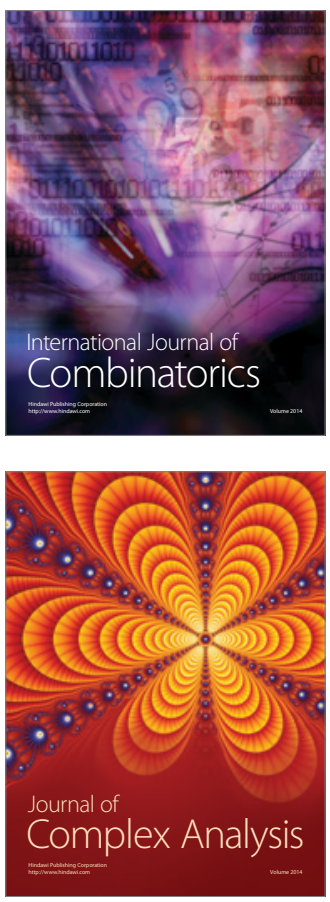

International Journal of

Mathematics and

Mathematical

Sciences
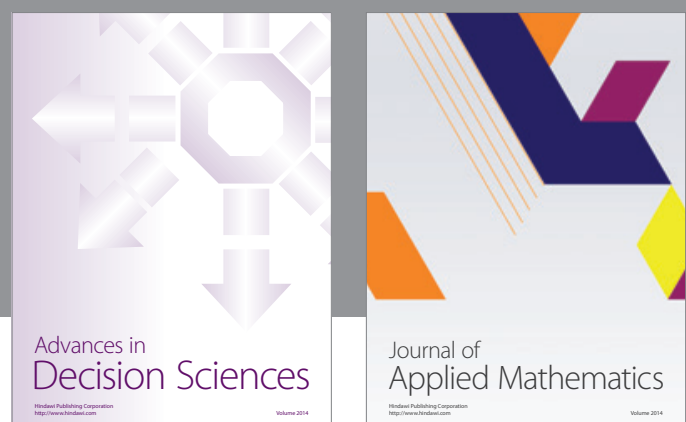

Journal of

Applied Mathematics
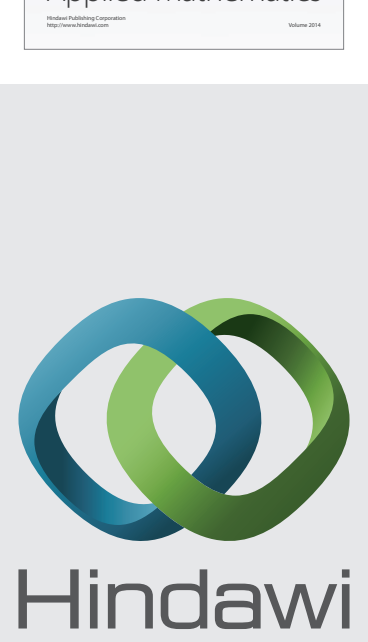

Submit your manuscripts at http://www.hindawi.com
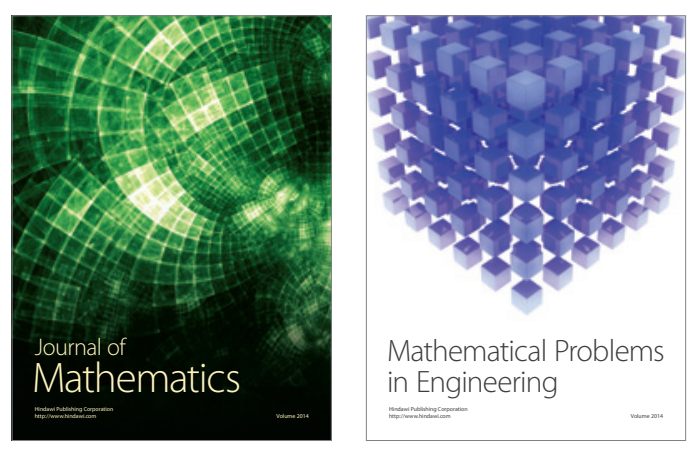

Mathematical Problems in Engineering
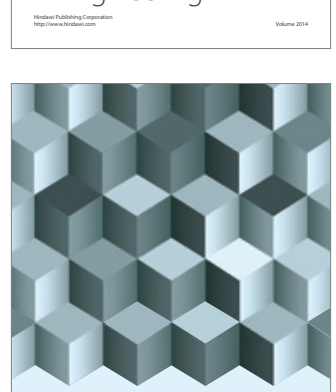

Journal of

Function Spaces
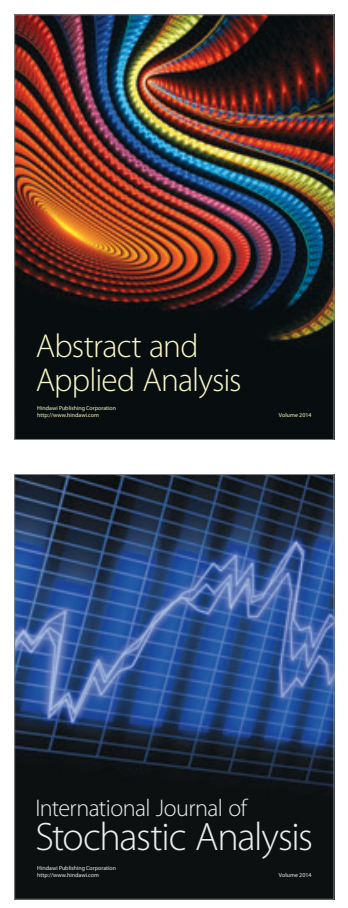

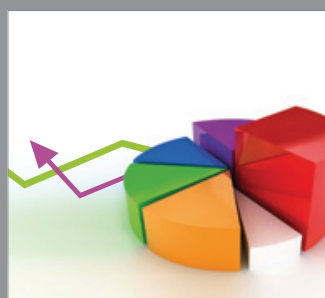

ournal of

Probability and Statistics

Promensencen
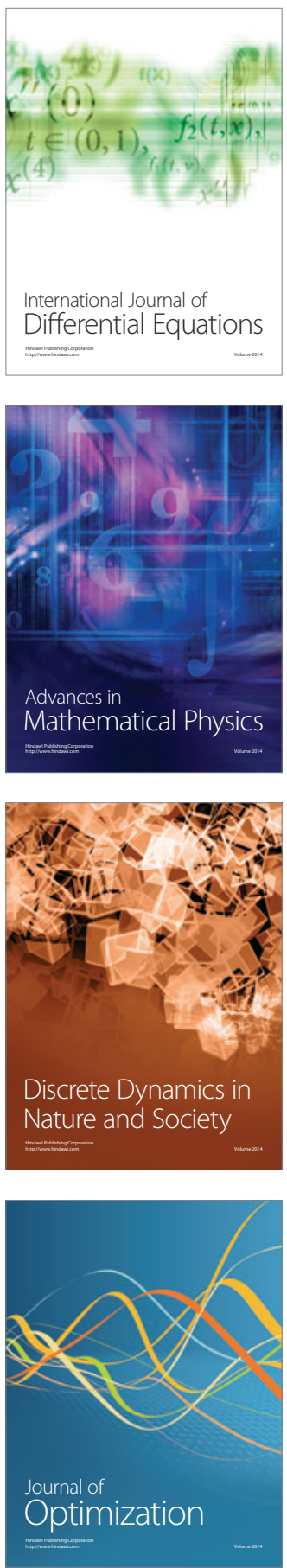